Dr. Gundlach: Nachträge zur Ornithologie Portorico's. 401

führt. Ob der Puffinus obscurus in Morris Birds of England derselbe ist?!

\title{
+4. Dysporus piscator.
}

Diese Art erhielt ich von meinem nun verstorbenen Freunde Don Gaspar Hernandez in Matanzas und da ich keine passende Beschreibung fand, nannte ich sie Dysp. Hernandezi Gundl. und wurde sie im Journal XXVI, 1878, Seite 298 bekannt gemacht. Im Jahre 1879 fand Mr. Ridgway, als er die Sula's des Smithsonian Institution bearbeitete, dass der sehr alte Vogel von Sula piscator dieselbe Färbung hat. Der Name Hernandezi ist nun Synonym.

\section{Nachträge zur Ornithologie Portorico's.} Von Or. Jean Gundlach.

Seit dem Erscheinen meiner „Neue Beiträge zur Ornithologie der Insel Portorico" Journ. Band XXVI, 1878, wurde die Liste der Arten vermehrt durch:

\section{Actiturus longicaudatus (Tringa) Lath. \\ 2. Greciscus jamaicensis (Rallus) Gmel.}

durch Dr. Stahl entdeckt und geändert Conurus Gundlachi Cab. anstatt Con. evops. Siehe Ornithologisches Centralblatt, Beiblatt zum Journal, VI, 1881, No. 1, p. 5.

\section{Tommaso Saivadori's Ornithologia della Papuasia e delle Molucche, Vol. II. Bericht von A. B. Meyer.}

Auf Seite 310-313 des Jahrganges 1880 unserer Zeitschrift hatten wir das Vergnügen, den ersten Band des obengenannten Werkes zur Anzeige zu bringen und wir befinden uns heute bereits in der glücklichen Lage, über das Erscheinen des zweiten Bandes berichten zu können. Dieser enthält auf 707 Seiten die Passeres und ist Herrn Francesco de Sanctis, dem Unterrichtsminister Italiens gewidmet, in Anerkennung dafür, dass derselbe den kostspieligen Druck des Werkes durch Unterstützung aus staatlichen Mitteln ermöglicht hat, während der erste Band in den Mem. der' R. Acad. d. Sc. di Torino erschienen und den Herren Beccari, d'Albertis, Bruijn und dem Marquis G. Doria Jeder in seiner Weise um die Arbeit verdient - zugeeignet war. 
Es wäre ungemein zu bedauern gewesen, wenn das Begonnene nicht hätte fortgeführt werden können, und es erscheint sehr auffallend, dass die Königliche Akademie der Wissenschaften in Turin die Gelegenheit hat vorübergehen lassen, ihre Momoiren durch die Drucklegung eines so wichtigen Werkes zu bereichern. Herr Salvadori ist nunmehr zum Theil auf die Subscription seitens der Fachgenossen angewiesen, und wir empfehlen daher dringend die Unterstützung des verdienstvollen Unternehmens. (Der 1. Band kostet 40 Lire, der 2. umfangreichere 50 - in Ansehung des Gebotenen sehr mässige Preise - die Ausstattung ist eine mustergültige).

Wie Seite 311, Jahrgang 1880 mitgetheilt, hat der Verfasser seinem Hauptwerke einen "Prodromus" vorausgesandt, und ist seitdem der X. Theil desselben in den Ann. Mus. Civ. di St. nat. di Genova, vol. XVI (1880) erschienen, enthaltend die Brachypodidae (3 Arten), Pittidae (11), Temeliidae (14), Saxicolidae (1), Sylvidae (10), Motacillidae (3), Ploceidae (12), Sturnidae (14), Oriolidae (7) und die Corvidae (9 Arten).

In dem vorliegenden zweiten Bande sind nicht weniger als 471 Arten abgehandelt, alle in der gleichmässig sorgfältigen und erschöpfenden Weise wie die 255 Arten des ersten Bandes. Diese 471 Arten vertheilen sich auf folgende 22 Familien und 128 Gattungen:

Hirundinidae (Seite 1-8) mit 4 Arten: Hirundo (3) und Hydrochelidon (1).

Muscicapidae (Seite 8-121) mit 115 Arten: Peltops (1), Pomarea (1), Monarcha (28), Arses (4), Sauloprocta (2), Rhipidura (24), Myiagra (9), Muscicapa (1), Erythrosterna (1), Megalestes (1), Monachella (1), Poerilodryas (9), Microeca (3), Gerygone (18), Aethomyias (1), Machaerorhynchus (3), Chenorhamphus (1), Clytomyias (1), Todopsis (5) und Malurus (1).

Campophagidae (Seite 121-167) mit 39 Arten: Grauculus (17), Edoliisoma (15), Lalage (5), Symmorphus (1) und Campochaera (1).

Artamidae (S. 167-173) mit 3 Arten der Gattung Artamus.

Dicruridae (Seite 173-184) mit 10 Arten: Dicruropsis (8), Dicranostreptus (1) und Chaetorhynchus (1).

Laniidae (Seite 184-239) mit 49 Arten: Cracticus (3), 
Salvadori's Ornitologia della Papuasia e delle Molucche. 403

Grallina (1), Rhectes (13), Colluricincla (5), Pachycephala (24), Pachycephalopsis (2) und Pachycare (1).

Menuridae (S. 239-241) mit 1 Art der Gattung Orthonyx.

Certhiidae (S. 241--242) mit 2 Arten: Climacteris (1) und Sittella (1).

Nectariniidae (S. 243-270) mit 17 Arten: Hermotimia (14) und Cyrthostomus (3).

Dicaeidae (Seite 270-290) mit 23 Arten: Dicaeum (15), Melanocharis (4), Urocharis (1), Pristorhamphus (1), Rhamphocharis (1) und Oreocharis (1).

Meliphagidae (S. 290-374) mit 89 Arten: Myzomela (19)*), Glycyphila (2), Conophila (1), Glycychaera (2), Oedistoma (1), Melilestes (4), Melipotes (1), Melidectes (1), Melirrhophetes (2), Meliarchus (1), Stigmatops (5), Ptilotis (11), Pycnopygius (1), Euthyrhynchus (3), Xanthotis (5), Melitograis (1), Philemonopsis (1), Philemon (7), Tropidorhynchus (1), Melithreptus (1) und Zosterops (19).

Brachypodidae (Seite $374-377$ ) mit 3 Arten der Gattung Criniger.

Pittidae (S. 377-404) mit 11 Arten der Gattung Pitta.

Timeliidae (Seite 405-418) mit 15 Arten: Melampitta (1), Brachypteryx (2), Serirornis (3), Pomatorhinus (1), Eupetes (5), Cinclosoma (1), Drymoedus (1) und Ortygocichla [im Nachtrage Seite 679] (1).

Saxicolidae (S. 418-429) mit 1 Art der Gattung Monticola. Sylviidae (S. 420-429) mit 10 Arten: Megalurus (2), Poodytes (1), Cisticola (3), Calamodyta (2), Locustella (1) und Phylloscopus (1).

Motacillidae (S. 430-433) mit 3 Arten: Budytes (1), Calobates (1) und Corydalla (1).

Ploceidae (S. 433-445) mit 12 Arten: Munia (6), Donacicola (3), Erythrura (1), Chlorura (1) und Neochmia (1).

Sturnidae (S. 445-471) mit 14 Arten: Sturnia (1), Calornis (6), Macruropsar (1), Lamprocorax (1), Basilornis (1), Melanopyrrhus (2) und Mino (2).

Oriolidae (Seite 471-482) mit 7 Arten: Mimeta (5) und Sphecotheres (2).

*) Seitdem beschrieb Herr Salvadori noch Myzomela erythromelas in Atti R. Ac. Sc. Tor. XVI, 8, 1881, von Neu-Britannien. 
404 Meyer: Salvadori's Orn. della Pap. e delle Molucche.

Corvidae (S. 483-493) mit 6 Arten: Corvus (4), Macrocorax (1) und Gymnocorax (1).

Paradiseidae (S. 494-678) mit 36 Arten: Lycocorax (3), Manucodia (5), Parotia (1), Lophorhina (1), Paradigalla (1), Astrapia (1), Epimachus (2), Drepanornis (2), Craspedophora (1), Seleucides (1), Semioptera (1), Paradisea (4), Uranornis (1), Diphyllodes (2), Schlegelia (1), Rhipidornis (1), Cicinnurus (1), Xanthomelus (1), Chlamydodera (1), Amblyornis (1) und Aeluroedus (4).

Unter diesen 22 Familien sind in Papuasien und den Molukken am reichsten die folgenden vertreten:

Muscicapidae mit 115 Arten in 20 Gattungen.

$\begin{array}{llllll}\text { Laniidae } & & 49 & & \text { in } 7 & 7 \\ \text { Meliphagidae } & 89 & & \text { in } 21 \\ \text { Paradiseidae } & 36 & \# & \text { in } 21 & \end{array}$

Am auffälligsten erscheint hier die weitgehende generelle Theilung bei den Paradiesvögeln: 36 Arten in 21 Gattungen, darunter 14 Gattungen mit nur je einer Art, 3 Gattungen mit je 2 Arten, 1 Gattung mit 3 Arten, 2 mit je 4 und 1 mit 5 Arten; und dieses in einer Gruppe, welche räumlich auf relativ so enge Grenzen beschränkt ist. Allerdings fügte Herr Salvadori den vielen vorhandenen Gattungen nur 2 neue hinzu: Uranornis und Rhipidornis, allein unseres Bedünkens hat hier die generelle Abspaltung innerhalb einer so natürlichen Gruppe wie die Paradiesvögel sie bildet, ihr höchstmögliches Maass erreicht; noch weitergehend würden Gattung- und Artbegriff sich decken, und damit an Sinn und Bedeutung verlieren.

Den Schluss des Bandes (S. 681-705) bildet wiederum ein willkommener Index mit nahezu 3000 Einträgen.

Wenn wir auch nicht annehmen dürfen, dass mit dem Abschluss des Werkes, nach Erscheinen des 3. Bandes, welchen der verehrte Verfasser auf die zweite Hälfte des Jahres 1882 verspricht, und welchem wir mit Verlangen entgegensehen, die Ornithologie der papuanischen Region für alle Zeit erschöpfend abgehandelt sei, da bei der Unbekanntschaft, in welcher wir uns in Betreff eines grossen Theiles von Neu-Guinea und vieler der umgebenden Inseln befinden, noch eine bedeutende Anzahl von Entdeckungen mit Sicherheit zu erwarten «steht, so legt doch diese mühselige Arbeit ein Fundament, auf welchem jederzeit 
mit Leichtigkeit weiter gebaut werden kann, so dass die gelehrte Welt Herrn Salvadori dauernd verpflichtet bleibt; und nur der eine Wunsch wird in uns rege: dass der Verfasser dereinst ein ähnliches Werk über die Avifauna der malayischen Region concipiren möge, da nur wenige Zeitgenossen über ein gleiches Maass an Kenntnissen und Ausdauer zu einer solchen Arbeit befähigt sein dürften.

\section{Beiträge zur Ornis des nördlichen Illinois.}

Von H. Nehrling.

(Fortsetzung; s. Seite 196-203).

\section{FAM.: FRINGILLIDAE. Finken.}

A. Subfamilie: Coccothraustinae. Gimpelfinken.

a) Genus Pinicola Vieill. Fichtengimpel.

64. Pinicola enucleator Cab. (Fichtengimpel, Kernbeisser, Tannengimpel, Pine Grosbeak). Kommt oft zahlreich in kalten schneereichen Wintern im nördlichen Illinois vor. Wie fast alle nordischen Vögel, so zeigt auch er bei seiner Ankunft durchaus keine Scheu, kommt zahlreich in die besonders mit Nadelholz bepflanzten Gärten und lässt sich leicht fangen. Manchmal erscheint er schon vor Weihnachten, gewöhnlich aber erst in den kalten Tagen des Januar. Oft ist er mehrere Winter nacheinander mit Aegiothus linaria, Loxia leucoptera, L. curvirostra americana, Plectrophanes nivalis, P. lapponicus und Ampelis garrulus eine der gewönlichsten Erscheinungen der Winterornis des nördlichen Illinois, oft fehlt er aber auch lange Jahre nacheinander. Er ist ein stattlicher Vogel und durch sein prachtvolles Roth im dunklen Grün der Tannen und Fichten eine prächtige Erscheinung. Die Beeren der Cedern, die Samen der Lebensbäume und Nadelhölzer bilden während dieser Zeit den Hauptbestandtheil seiner Nahrung. Schon zeitig im März zieht er wieder hinauf in die arktischen Regionen. Viele werden in Winter gefangen, doch scheint ihnen die Hitze der Sommermonate nicht zuzusagen, denn sie sind dann still und ruhig und erst mit Eintritt kälteren Wetters gewinnen sie ihre frühere Lebhaftigkeit wieder. 


\section{$2 \mathrm{BHL}$ Biodiversity Heritage Library}

1881. "Tommaso Saivadori's Ornithologia della Papuasia e delle Molucche, Vol. II." Journal

$f u$

r Ornithologie 29, 401-405. https://doi.org/10.1007/bf02002534.

View This Item Online: https://www.biodiversitylibrary.org/item/102782

DOI: https://doi.org/10.1007/bf02002534

Permalink: https://www.biodiversitylibrary.org/partpdf/142852

\section{Holding Institution}

Smithsonian Libraries

\section{Sponsored by}

Biodiversity Heritage Library

\section{Copyright \& Reuse}

Copyright Status: Public domain. The BHL considers that this work is no longer under copyright protection.

This document was created from content at the Biodiversity Heritage Library, the world's largest open access digital library for biodiversity literature and archives. Visit BHL at https://www.biodiversitylibrary.org. 